\title{
The Simulation Model of the Asynchronous Trans- formation of Self-Similar Traffic in Switching Nodes Using a Queue
}

\author{
Gennadiy I. Linets \\ North-Caucasus Federal University \\ Stavropol, Russian Federation \\ kbytw@mail.ru
}

\author{
Svetlana V. Govorova \\ North-Caucasus Federal University \\ Stavropol, Russian Federation \\ mitnik2@yandex.ru \\ Valeriy P. Mochalov \\ North-Caucasus Federal University \\ Stavropol, Russian Federation \\ mochalov.valery2015@yandex.ru
}

\author{
Roman A. Voronkin \\ North-Caucasus Federal University \\ Stavropol, Russian Federation \\ roman.voronkin@gmail.com
}

\begin{abstract}
The simulation model of the asynchronous transformation of self-similar packets flow in the switching nodes has been developed. The producer-consumer pattern with a limited-sizes queue was used for the model; the asynchronous programming method was used to transform the self-similar traffic in the switching nodes. Unlike the wellknown queuing models, the developed simulation model allow to take into account a limited amount of buffer memory when servicing a self-similar packets flow. The simulation model allows to investigate: the dependency between the size of the buffer and the network performance in terms of the quality of service; the amount of lost packets depending on the queue size; the effect of the queue size on the number of processed packets; the dependence of the number of lost packets on time with different Hurst exponent values.
\end{abstract}

Keywords-Ttelecommunication network, self-similar traffic, Hurst index, performance, producer-consumer, asynchronous conversion, Pareto distribution.

\section{INTRODUCTION}

One of the problems of transport telecommunication networks is the effective use of hub and channel resources when servicing self-similar traffic. Its solution requires the development of new methods to improve performance by optimizing network resources. The analysis of the used traffic models of modern and prospective networks to determine the probability-time characteristics of the information exchange of users leads to the conclusion that the use of Poisson models in many cases leads to an underestimation of the load and, consequently, to the impossibility of providing the required indicators of the Quality of Service (QoS) [1-3]. It is because the properties of computer and voice traffic have significant differences, the main of which is the presence of "long memory" in the distribution of the lengths of busy computer traffic that has "heavy tails" and is well characterized by Hurst exponent $\mathrm{H}$.

The important parameter of the information exchange affecting the quality of service and network performance, is the losing messages probability due to the overflow of buffer storage devices (RAM) at switching nodes.

The queuing systems with self-similar traffic are being paid fairly close attention [2, 4-7]. However, the obtained results do not allow to cover the whole variety of traffic properties of modern applications. In the most of works for example [1, 7-9] it is shown that the selfsimilarity phenomenon in modern networks manifests itself asymptotically at large time intervals and depends on various reasons, one of which is that the information processing technologies used imply the transmission over one channel with different performance requirements. As a result, fractality and heterogeneity are manifested in its properties. In [10,11], it was concluded that with increasing self-similarity, it is necessary to increase the buffer size to reduce the likelihood of packet loss. However, an unjustified increase in the buffer space leads to the decrease of the quality of information exchange, for example, to an increase in the average delay.

The simulation model of asynchronous transformation of self-similar traffic based on the producerconsumer pattern with a limited buffer in the switching nodes has been presented in the article. Also the lowest limit of the buffer memory is determined depending on the Hurst exponent value, when the packet loss probability equals to zero.

\section{THE PROBLEM ANALYSIS}

In transport networks, the buffer for arriving packages has a limited volume. When a large number of packets are received at the input of the system, the blocking may 
occur, i.e. out of service packages are lost as a result. The number of lost packets is an indication of the quality of service. There are a lot of issues concerned with improving the performance of computing systems and networks and proposed models for solving this problem. In the article $[11,12]$ the models are described for calculating QoS in next-generation networks, focusing on the traffic properties of IP-based multi-service networks and the influence of end devices on the main indicators of service quality - delays and losses. However, an effective method of obtaining high network performance while maintaining self-similar traffic and satisfying QoS requirements in these works was not proposed. In [1-3], the issues of optimizing the buffer memory volume of switching nodes during the transmission of self-similar traffic were considered. A block diagram of a device for converting arrivals traffic, which allows the conversion of an arbitrary distribution of time intervals between packets to a Poisson law, is presented. The disadvantage of this model is the presence of two elements of the buffer memory, which ensure the cyclical nature of the functional transformations of the input self-similar flow of packets.

\section{THE STATEMENT OF THE PROBLEM}

In order to improve the performance of modern information and computing networks, it is necessary to develop a simulation model that transforms the arrival packets flow, which is self-similar $G\left(\tau_{1}\right)$, into a given distribution law $G\left(\tau_{2}\right)$, in particular, into the Poisson or exponential one. The transformation object is a onedimensional density of the distribution of time intervals between packets of the arrivals flow $G\left(\tau_{1}\right)$.

Let's determine the requirements for the simulation model. The simulation model should aim at investigating:

- the effect of buffer size $M$ on network performance;

- the number of lost packets depending on the size of the queue;

- the dependence between the number of lost packets on time and Hurst exponent values.

The restrictions for a simulation model are:

- the limited buffer size of the switching node M;

- the buffer uses FIFO servicing discipline.

- It is necessary to obtain:

- the number of lost packages depending on the queue size and the Hurst exponent value;

- QoS estimation depending on the buffer size which used for servicing self-similar traffic;
- the dependence between buffer size and Hurst exponent in case of lossless servicing.

\section{THE PROBLEM SOLUTION}

The imitation model is based on the producer / consumer pattern, which is used in asynchronous programming. It means one or several tasks "producer" data asynchronously and one or several tasks "consuming" them. The non-triviality of the problem lies in the fact that, potentially, both the creation of new data and their consumption may take a long time. Based on the conditions of the problem being solved, it is required that the data should be processed as quickly as possible.

The "producer-consumer" relationship implies a oneway communication channel used for transmitting packets. For this purpose, processes are linked through a buffer. The arrived packets are not consumed immediately, but should be able to be enqueued. The buffer works using the FIFO service discipline. The task of "producerconsumer" is to provide concurrent access of the input and output data flow to the shared buffer.

In the article, a limited-sized buffer is used. A selfsimilar packet flow arrives at the system input (the Hurst exponent value $\mathrm{H}>0.5$ ), and the output is a packet flow whose distribution density of time intervals between packets is distributed according to the exponential law. The input and output streams are matched by the value of the expectation.

The block diagram of the model for traffic transform is shown in figure 1 .

To implement the simulation model, the programming language Python 3.7 and the packages NumPy, SciPy, and matplotlib were used. Also Jupyter Notebook was used as a tool for creating analytical reports, which allows you to save together program code, images, comments, formulas and graphs [13].

The simulation model work consists of several steps:

1. Setting the number of generated packets (population size) $\mathrm{N}$ and queue size $\mathrm{M}$.

2. The definition of "producer" and "consumer" coprocedures for launching in the event loop.

3. The objects creation. The description of a random variable is distributed according to a given law (in our case it is Pareto distribution).

4. The time intervals sequence between packets generation. For this aim, we need a Hurst exponent value and a random generator seed.

5. Modeling the process of converting a stream of packets by means of asynchronous programming. 


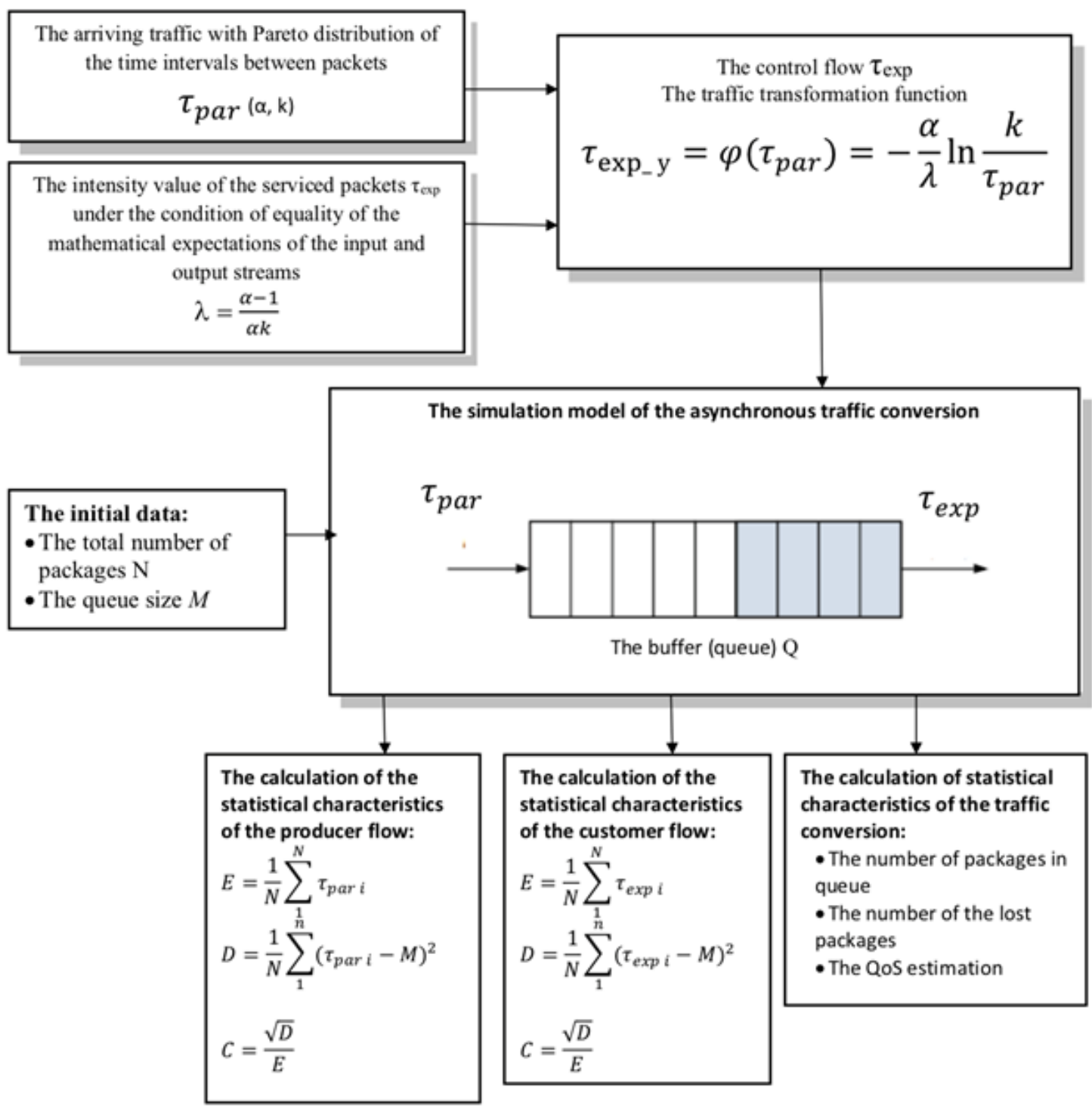

Fig 1. The block diagram of the model for traffic transform

6 . The calculation of the expectation (E) of a random variable describing the time intervals between arriving packages.

7. The object creation. It is required to obtain an exponential packet flow with an expectation equal to the expectation of the arrived packets flow. The control sequence is generated in the same way as the input stream.
8. Running a simulation of the packet stream transformation process.

9. The calculation of the statistical characteristics for the serviced packets flow: the expectation (E), the variance (D).

The UML sequence diagram describing the work of the developed model is shown on figure 2 . 


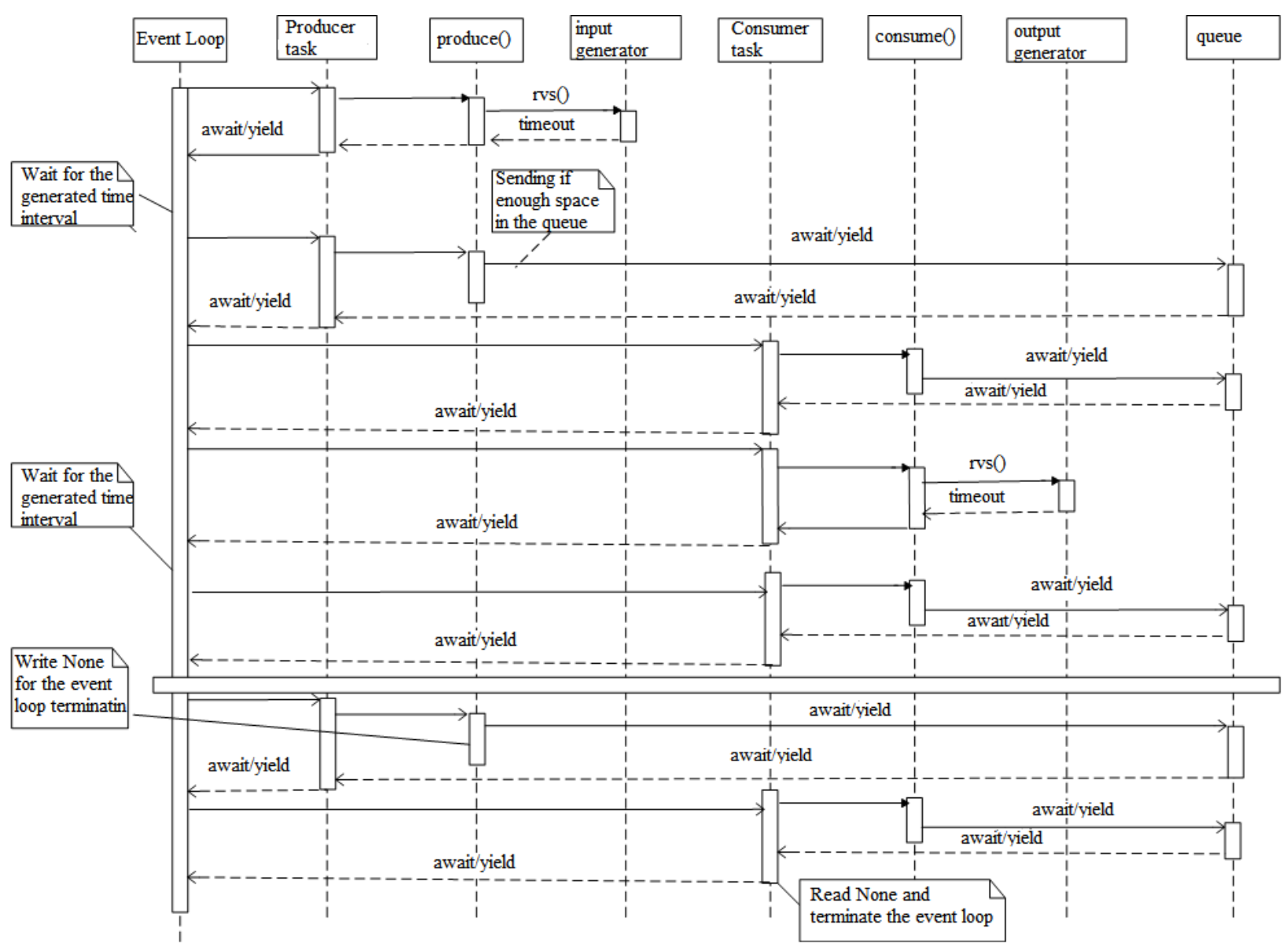

Fig. 2. The UML sequence diagram describing for the developed model

\section{EXAMPLE}

Consider the use of a simulation model for the analysis of indicators of the quality of information exchange.

The initial data:

- $\quad$ the population size $\mathrm{N}=1000$;

- $\quad$ the queue size $M=10,50,100$;

- the time interval distribution between packets is Pareto;

- the packet flow service is implemented according to FIFO.

It is required to obtain:

- the number of lost packages depending on the queue size and the Hurst exponent value;

- QoS estimation depending on the buffer size which is used for servicing self-similar traffic;

- the dependence between buffer size and Hurst exponent in case of lossless servicing.
The simulation results are presented in the table 1 and on the figures 3-7. On the table 2 and the figure 8 is shown the dependency between buffer size $M$ and Hurst exponent $H$ for the case of servicing the self-similar packets flow with lossless.

\section{CONCLUSION}

Thus, it is possible to draw the following conclusions:

1. Using a fixed volume of buffer memory, the value of packet loss increases with the increasing Hurst exponent.

2 . When we increase buffer memory volume, the amount of successfully processed packets increases in the case of the fixed Hurst exponent value $\mathrm{H}$.

3. With an increase in the Hurst exponent value $H$, the network performance decreases sharply, and, the higher the Hurst exponent, the lower the network performance.

4. The analysis of the lost packets amount in time has shown that the larger the buffer volume and the smaller the Hurst exponent value, the smaller the packet loss. 
TABLE I. THE STATISTICAL CHARACTERISTICS OF THE DEVELOPED MODEL

\begin{tabular}{|c|c|c|c|c|c|c|c|c|c|}
\hline \multirow{3}{*}{$\begin{array}{c}\text { The Hurst } \\
\text { exponent } \\
\text { value, } H\end{array}$} & \multicolumn{9}{|c|}{ The statistical characteristics } \\
\hline & \multirow{2}{*}{$\begin{array}{l}\text { The arrived packets } \\
\text { flow } \\
\text { Mean, } E\end{array}$} & \multicolumn{2}{|c|}{ The control flow } & \multicolumn{3}{|c|}{ The packets flow of the "producer" } & \multicolumn{3}{|c|}{$\begin{array}{c}\text { The packets flow of the } \\
\text { "consumer" }\end{array}$} \\
\hline & & Mean, $E$ & Variance, $D$ & Mean, $E$ & $\begin{array}{c}\text { Variance, } \\
D\end{array}$ & $\begin{array}{c}\text { Coefficient of } \\
\text { variation }\end{array}$ & $\begin{array}{c}\text { Mean, } \\
E\end{array}$ & Variance, $D$ & $\begin{array}{c}\text { Coefficient of } \\
\text { variation }\end{array}$ \\
\hline 0.6 & 2.25 & 2.25 & 5.06 & 2.11 & 2.04 & 0.97 & 2.38 & 2.39 & 1.00 \\
\hline 0.8 & 3.50 & 3.50 & 12.25 & 2.89 & 4.50 & 1.55 & 3.72 & 3.74 & 1.00 \\
\hline 0.95 & 10.99 & 10.99 & 120.99 & 4.65 & 12.15 & 2.60 & 11.81 & 11.35 & 0.96 \\
\hline
\end{tabular}

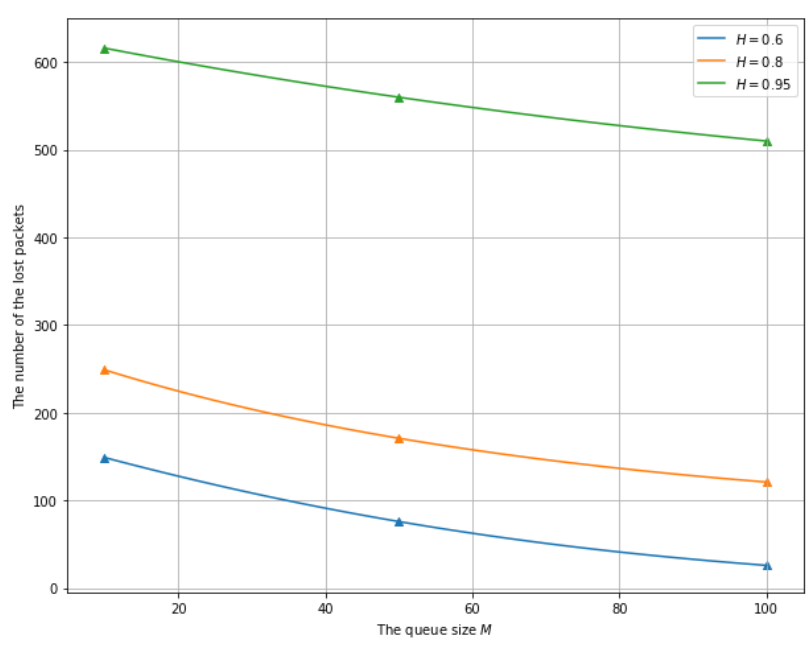

a)

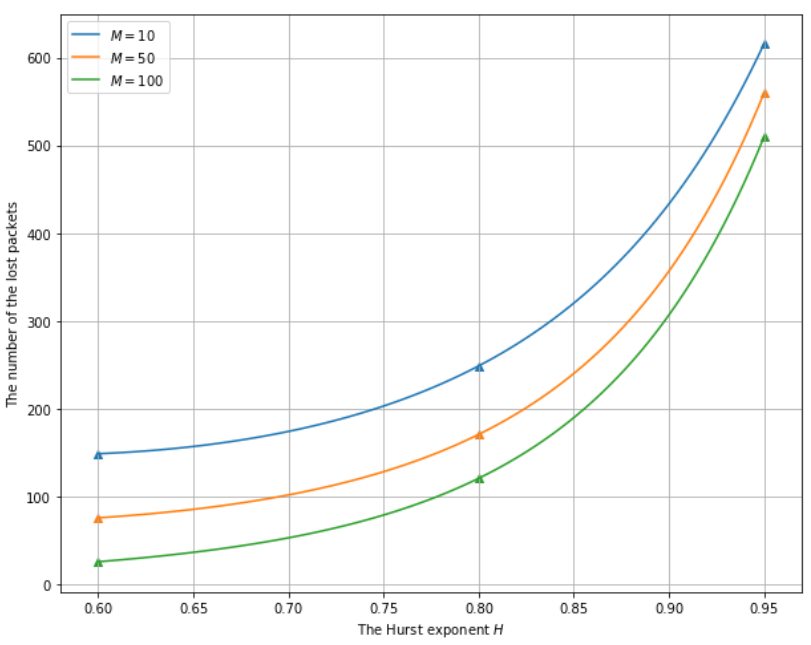

b)

Fig. 3. The number of the lost packages in the dependence on: a) the queue size $\mathrm{M}$; b) the Hurst exponent $\mathrm{H}$

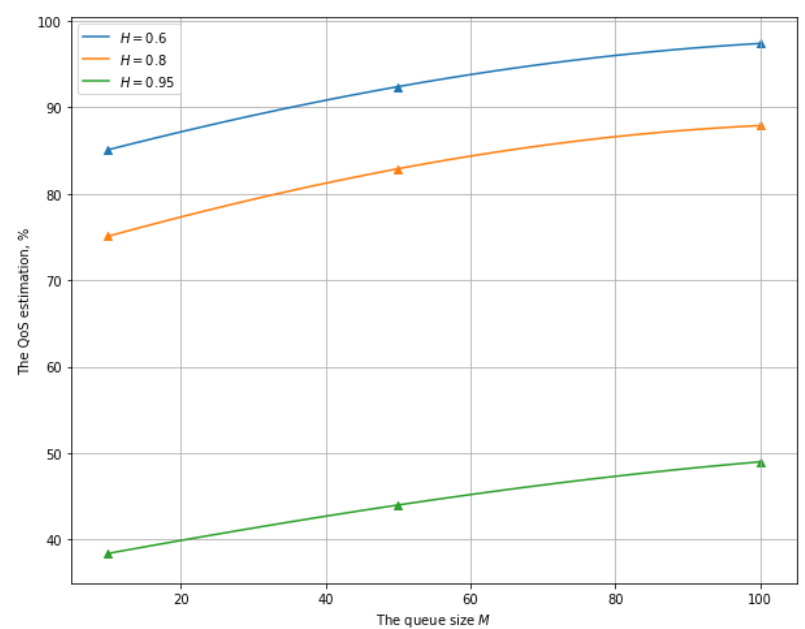

a)

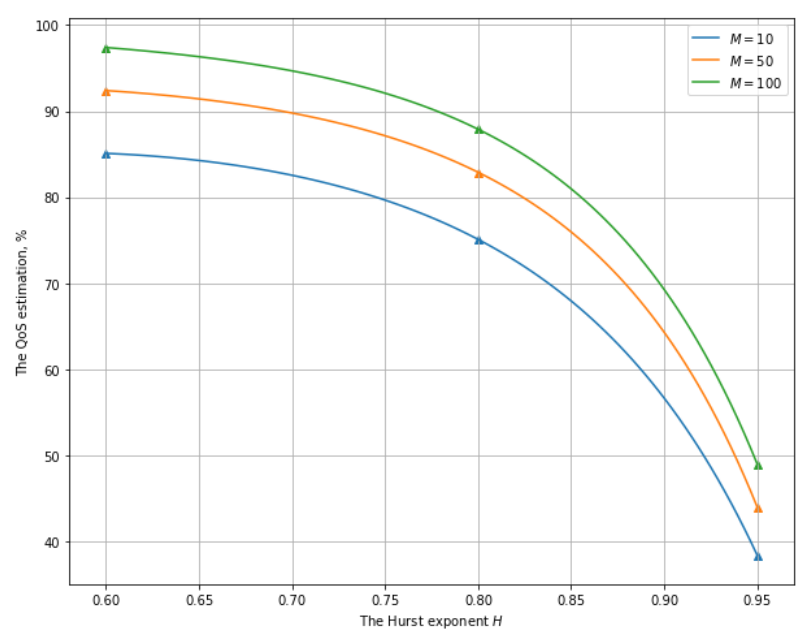

b)

Fig. 4. The QoS estimation in the dependence on: a) the queue size M; b) the Hurst exponent $\mathrm{H}$ 


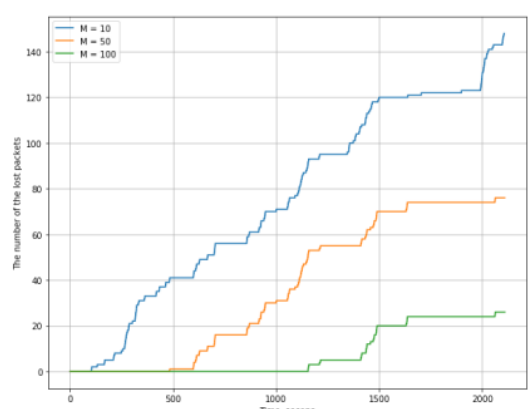

a)

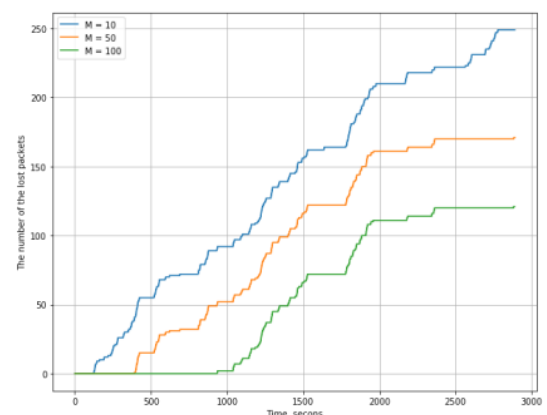

b)

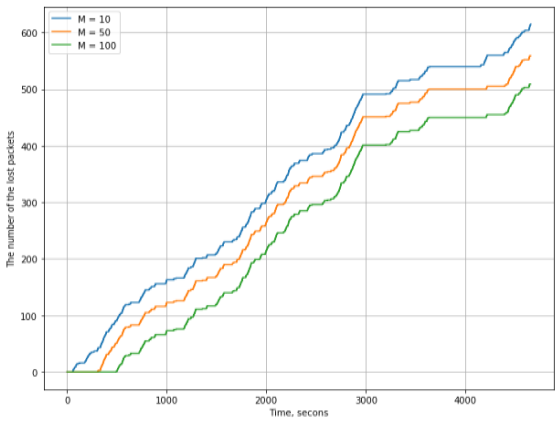

c)

Fig. 5. The number of the lost packages in time for the fixed buffer sizes $(M=10, M=50, M=100)$ and the Hurst exponent values: a) $H=0.6$; $b) H=0.8$; $c)$ $\mathrm{H}=0.95$

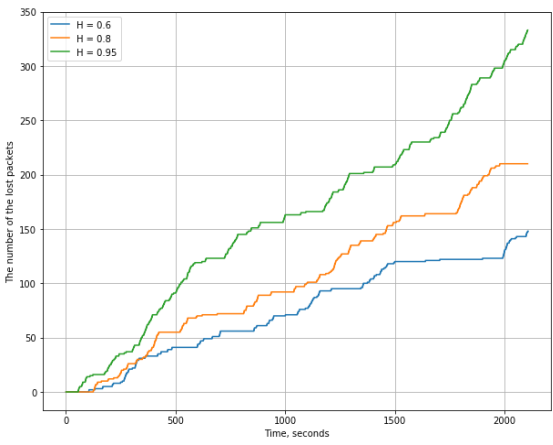

a)

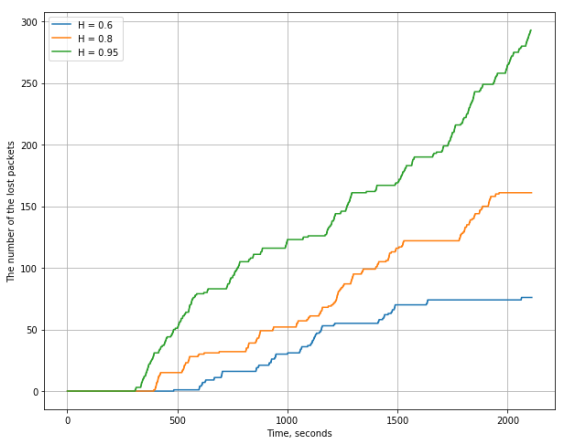

b)

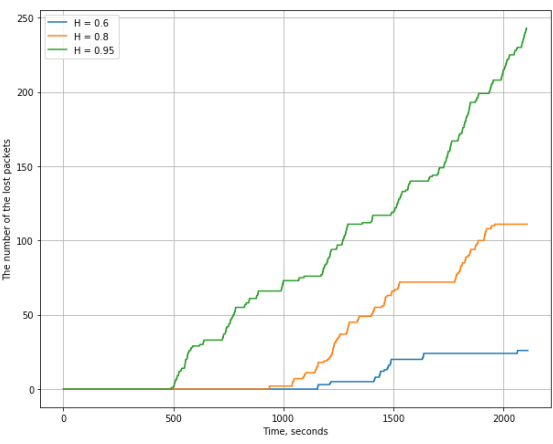

c)

Fig. 6. The number of the lost packages for the fixed Hurst exponent values $(H=0.6, H=0.8, H=0.95)$ and the buffer sizes: a) $M=10 ; b) M=50 ; c) ~ M=100$

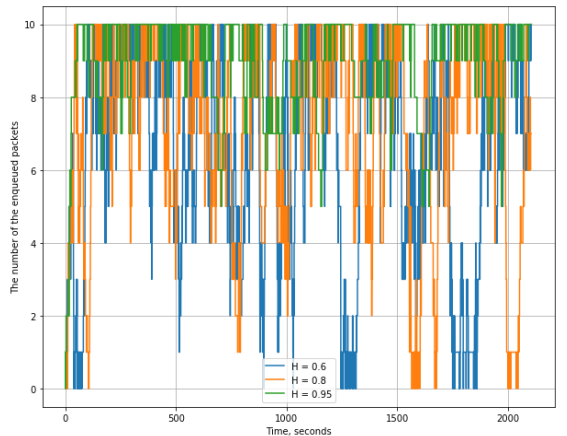

a)

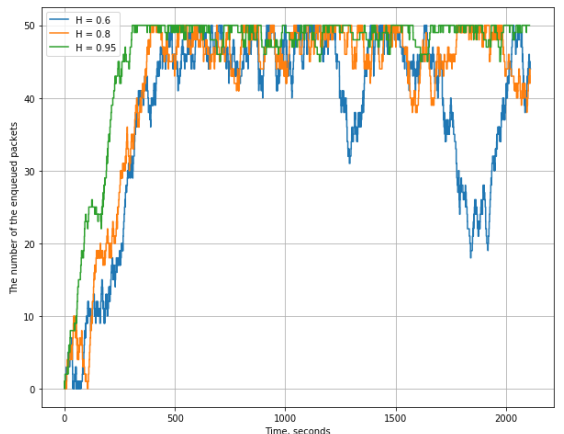

b)

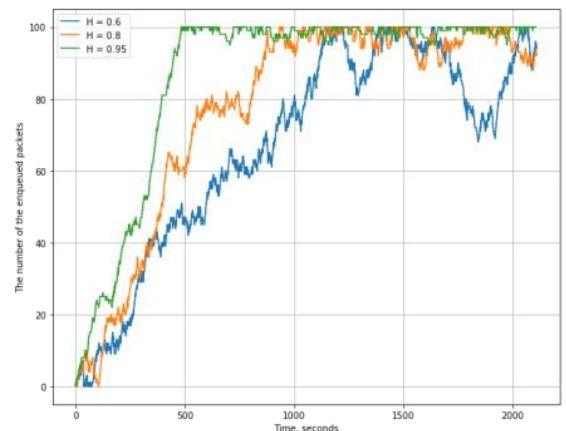

c)

Fig. 7. The number of the enqueued packages for the fixed Hurst exponent values $(H=0.6, H=0.8, H=0.95)$ and the buffer sizes: a) $M=10$; $b) \mathrm{M}=50$; $c)$ $\mathrm{M}=100$

5. Using a fixed buffer size, the number of packets in the queue is higher, the larger the Hurst exponent value.

6. The lowest limit of the buffer size $\mathrm{M}$ is determined while servicing the self-similar flow for different Hurst exponent values within lossless servicing.
Basing on the research we can draw the following conclusion: while servicing a self-similar packet flow, it is necessary to simultaneously control the volume of buffer memory and the bandwidth of switching nodes to meet the required QoS estimation for network users. 
TABLE II. THE BUfFER SIZE M VALUES IN DEPENDENCY OF THE HURST EXPONENT VALUES H

\begin{tabular}{|c|c|}
\hline Hurst exponent $\boldsymbol{H}$ & Buffer size $\boldsymbol{M}$ \\
\hline 0.55 & 115 \\
\hline 0.6 & 126 \\
\hline 0.65 & 139 \\
\hline 0.7 & 160 \\
\hline 0.75 & 186 \\
\hline 0.8 & 221 \\
\hline 0.85 & 299 \\
\hline 0.9 & 407 \\
\hline 0.95 & 609 \\
\hline
\end{tabular}

\section{ACKNOWLEDGEMENT}

The investigation was carried out with the financial support of the Russian Foundation for Basic Research as a part of the research project No. 19-07-00856\19.

\section{REFERENCES}

[1] Linets G.I.: Methods of structural and parametric synthesis, identification and management of transportation telecommunication networks for maximum performance. Monograph. - Stavropol, 2011. $-384 \mathrm{p}$.

[2] Rajaiah Dasari, Ramesh Renikunta, Malla Reddy Perati, SelfSimilar Network Traffic Modelling Using Fractal Point ProcessMarkovian Approach, Contributions from the International Conference and Workshop on Fractals and Wavelets: Fractals, Wavelets, and their Applications, Switzerland, 2014, pp 413-425.

[3] Linets G. I., Fomin L .A., Govorova S. V. Device for traffic conversion. Patent for invention № 2591649, application № 2015111937, registration in the state register of inventions of the Russian Federation 22.06.16.

[4] Dmytro A., Nameer Qasim. LTE EPS Network with Self-Similar Traffic Modeling for Performance Analysis. Second International Scientific-Practical Conference: Problems of Infocommunications Science and Technology, 2015, pp. 275-277.

[5] Mikova S. Iu., Oladko V. S., Nesterenko M. A. Podkhod k klassifikatsii anomalii setevogo trafika [The approach to classification of anomalies in network traffic]. Innovatsionnaia nauka, 2015, no. 11, pp. 78-80 (in Russian).

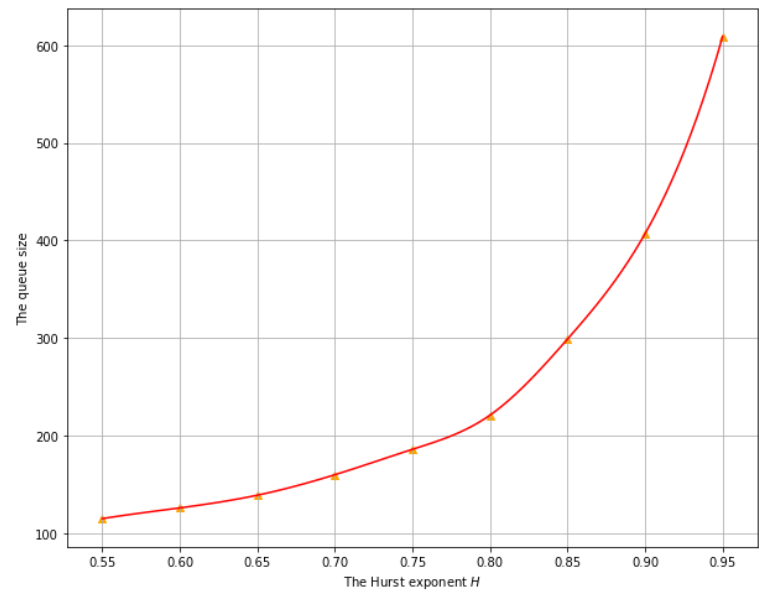

Fig. 8. The buffer size $\mathrm{M}$ graph in dependency of the Hurst exponent value $\mathrm{H}$

[6] Kleinrock L. Communication networks (stochastic flows and delay messages) / per.from English. M.: Science, 1970. 256 p.

[7] Sheluhin O.I., Smolskiy S.M., Osin A.V. Self-similar processes in telecommunications. - John Wiley \& Sons, 2007.

[8] Chengyu Zhu and Oliver W. W. Yang, "A Comparison of Active Queue Management Algorithms Using the OPNET Modeler", IEEE Communications Magazine, June 2002

[9] Ranadheer Donthi, Ramesh Renikunta, Rajaiah Dasari, Malla Reddy Perati, Self-Similar Network Traffic Modeling Using Circulant Markov Modulated Poisson Process. Contributions from the International Conference and Workshop on Fractals and Wavelets: Fractals, Wavelets, and their Applications, Switzerland, 2014, pp 437-444.

[10] F. Ferreira (speaker) and A. Pacheco, "Analysis of Pareto Type/M/s/c finite buffer queues.", Second Workshop on New Trends in Modelling, Quantitative Methods and Measurements, Aveiro, Portugal, 24/11/2005 - 25/11/2005.

[11] Miloucheva I., Müller E., Anzaloni A., A practical appoach to forecast Quality of Service parameters considering outliers. 2003.

[12] Linev A.V., Kudin A.V Arhitektura i operacionnie sistemi parallelnih vichislitelnih system [Architecture and operating systems of parallel computing systems]. N. Novgorod, NNGU, 2007. $72 \mathrm{p}$.

[13] Linets G.I., Govorova S.V., Voronkin R.A. Simulation model of asynchronous conversion of self-similar traffic using a queue. Certificate of State. registration of computer programs № 2019610440. Date register. 01/10/2019. 